\title{
Zur Frage der Existenz definierter Hydrate in Natronlaugen verschiedener Konzentration und deren Einfluß auf die Bildung von Cellulosexanthat
}

\author{
von Kurt Hess *) \\ Technische Hochschule Hannover
}

In der kürzlich erschienenen Abhandlung von R. B a rtunek (1) wird für Reaktionen zwischen Cellulose als Bodenkörper und Elektrolytlösungen die Aufmerksamkeit auf die Bedeutung des Hydratationszustandes der reagierenden Komponente in diesen Lösungen gelenkt, wobei vornehmlich Versuche angegeben werden, die

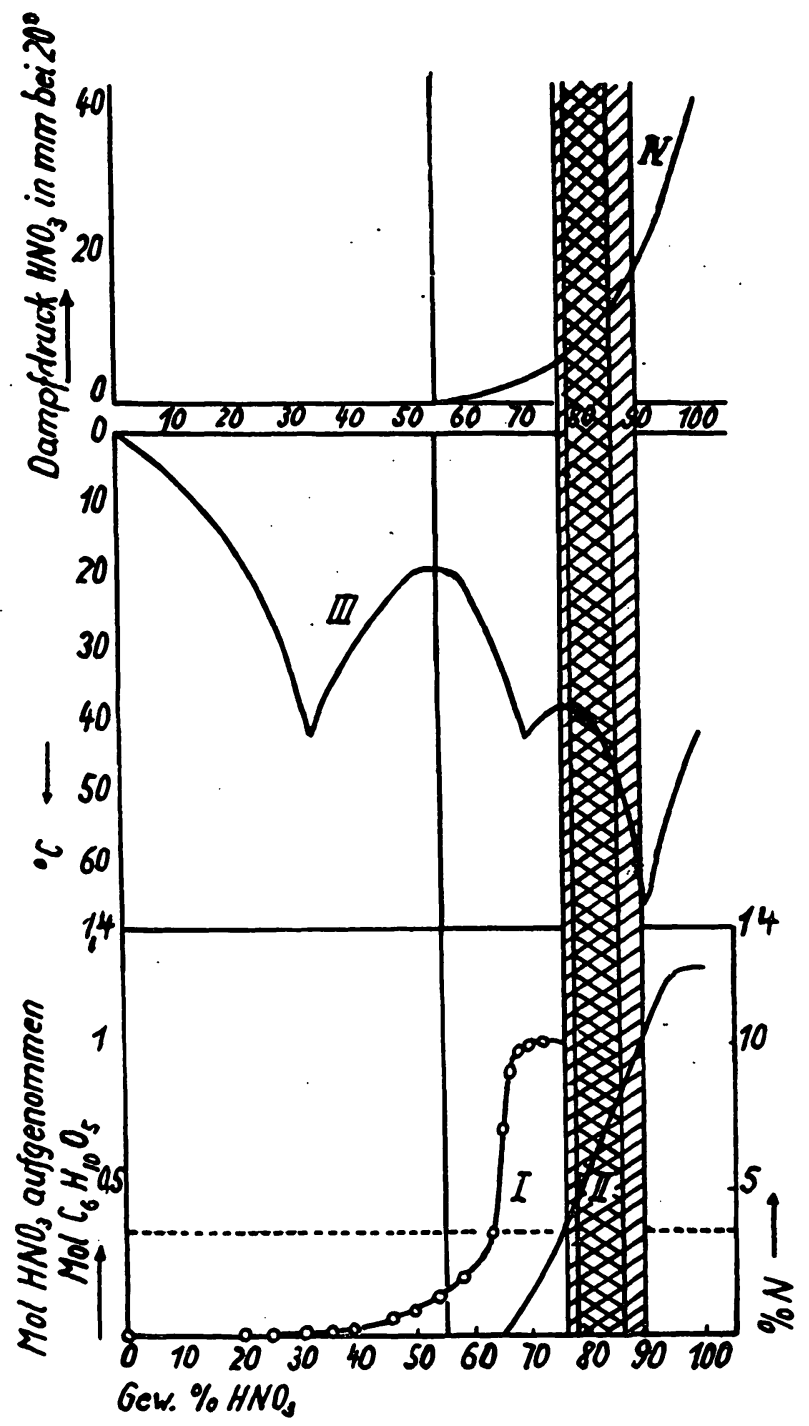

Abb. I. Bildung der Knecht-Verbindung (Kurve I); Stickstoffaufnahme der Cellulosefaser (Kurve II); Gefrierpunkt der Salpetersäure nach Küster-Kremann (Kurve III); Partialdruck der freien Salpetersäure bei $20^{\circ}$ nach Taylor (Kurve IV) bei wässeriger Salpetersäure in Abhängigkeit von der Säurekonzentration; schraffierter Streifen: starke Faserquellung; doppelt schraffierter Streifer : Auflösung der Faser in der Säure.

*) Vgl. auch die Diskussionsbemerkung v. K. Hess nach dem Vortrag von H. Bartunek auf der Hauptversammlung des Vereins Zellchèming Juni 1954 in Baden-Baden. diese Bedeutung für die Alkalisierung und Xanthogenierung der Cellulose mit Natronlauge und Schwefelkohlenstoff begründen sollen.

Es besteht kein $\mathrm{Zweifel,} \mathrm{daß} \mathrm{bei} \mathrm{einer} \mathrm{allseitigen} \mathrm{Be}-$ schreibung einer Reaktion in wäßrigen und nicht wäßrigen Systemen der Hydratations- bzw. Solvatationszustand der Komponenten berücksichtigt werden muß. Daß dies auch bei der Umsetzung von Cellulosefasern mit Elektrolyten gelegentlich zweckmäßig sein kann, ist von C. Trogus (2) in einer Arbeit aus dem Laboratorium von K. Hess 1934 am Beispiel der Wechselwirkung von Salpetersäure mit Cellulose gezeigt worden.

Abb. 1 gibt jeweils die Abhängigkeit der von der Salpetersäure-Konzentration zusammengestellten Eigenschaften im System Cellulose $/ \mathrm{HNO}_{3} / \mathrm{H}_{2} \mathrm{O}$, und zwar Kurve I den Existenzbereich der Doppelverbindung zwischen Cellulose und $\mathrm{HNO}_{3}$, die durch Analyse und das charakteristische Röntgen-Faserdiagramm leicht gekennzeichnet werden kann (,Knechtverbindung " der Cellulose mit der Zusammensetzung $1 \mathrm{Mol} \mathrm{C}_{6} \mathrm{H}_{10} \mathrm{O}_{5}$ : $1 \mathrm{HNO}_{3} ; \mathrm{HNO}_{3}$ leicht mit Wasser auswaschbar). Kurve II gibt den Nitrostickstoff-Gehalt der gebildeten Nitrocellulose, Kurve III den. Gefrierpunkt der wäßrigen Salpetersäuren nach Küster-Kremann, durch die die Existenz zweier verschiedener Hydrate $\left(\mathrm{HNO}_{3}-\right.$ Hydrate) erwiesen werden, und Kurve IV den Partialdruck der freien, nicht hydratisierten Salpetersäure bei $20^{\circ}$ nach T a ylor. Durch Vergleich der Kurven I bis IV ergibt sich, daß der Existenzbereich der Knechtverbindung im Existenzbereich des Monohydrates der Salpetersäure liegt, daß im Existenzbereich des Trihydrates weder Bildung der Knechtverbindung noch Nitrierung eintritt und daß die Nitrierung gemäß der Dampfdruckkurve für wasserfreie $\mathrm{HNO}_{3}$ und nicht durch eines der beiden Hydrate erfolgt.

Die Übertragung einer derartigen Zuordnung von verschiedenartigen Reaktionsprodukten $\mathrm{zu}$ definierten Hydratationsstufen des Reagens auf das System Cellulose $/ \mathrm{NaOH} / \mathrm{H}_{2} \mathrm{O} / \mathrm{CS}_{2}$ ist vorerst schwierig, vor allem deshalb, weil die Existenz verschiedener Hydrate für Natronlaugen bisher überhaupt noch nicht erwiesen ist.

Um trotzdem den Einfluß der $\mathrm{NaOH}-$ Konzentration auf Alkalisierung und Sulfidierung festzulegen, wird von R. Bartunek Schwefelkohlenstoff auf eine Cellulosefaser-Suspension in Natronlaugen steigender Konzentrationen ( 2 bis $44 \%$ ) unter Umwälzen in rotierenden Zylindern bei konstanter Zeit (360 Min.) und Temperatur $\left(20^{\circ} \mathrm{C}\right)$ zur Einwirkung gebracht und der sich dabei lösende Celluloseanteil als Maß für die Wirkung der Natronlauge bestimmt. Der Verlauf der in Abhängigkeit von der Laugenkonzentration gelösten Cellulosemenge ist in Kurve II der Abb. 2 dargestellt. Kurve I in $\mathrm{Abb} .2$ gibt unter gleichen Bedingungen die $\mathrm{CS}_{2}-$ 


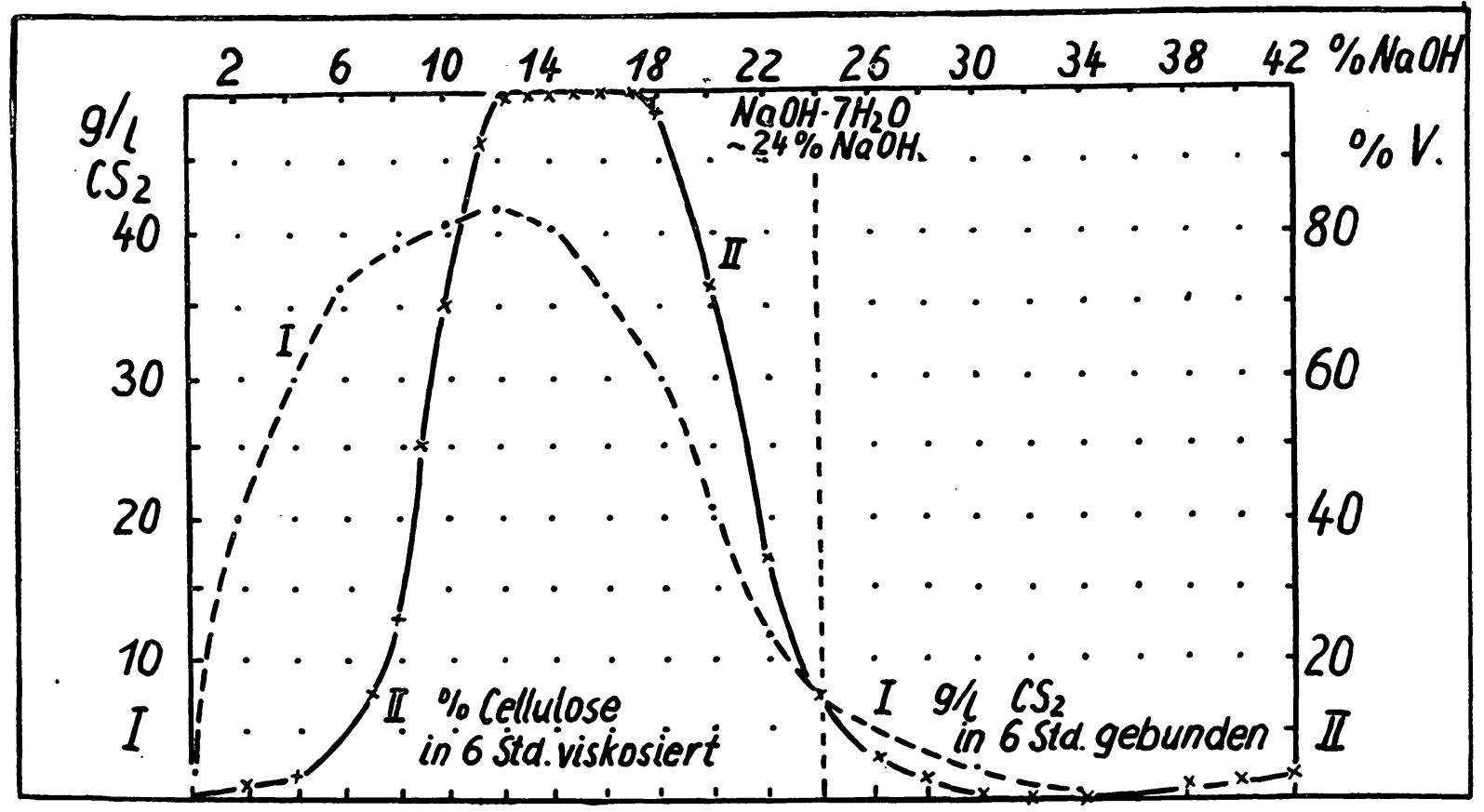

Abb. 2. System $\mathrm{NaOH}-\mathrm{H}_{2} \mathrm{O}-\mathrm{CS}_{2}$ und $\mathrm{NaOH}-\mathrm{H}_{2} \mathrm{O}-$ Cellulose $-\mathrm{CS}_{2}$ (nach $\mathrm{H}$. Bartunek).

Kurve I $\mathrm{CS}_{2}$-Bindung durch Natronlaugen steigender Konzentration;

Kurve II Lösbarkeit von Cellulose durch $\mathrm{CS}_{2}$ in Natronlaugen steigender Konzentration

Aufnahme durch die Laugen bei Abwesenheit von Cellulose.

Der Wendepunkt in den Kurven und ihre Ähnlichkeit im absteigenden Ast veranlassen nun in den Veröffentlichungen von Bartunek die Annahme, daß in den Natronlaugen mit Konzentrationen unterhalb des Wendepunktes andere Hydrate mit anderen Reaktionsweisen vorliegen als in den Laugen oberhalb, und daß sich diese Hydrate in vergleichbarer Weise sowohl auf die Reaktion zwischen $\mathrm{CS}_{2}$ und Natriumhydroxyd als auf die Xanthogenierung, nämlich „stark hemmend“, auswirken (,Reaktions-Wendepunkte" "); in der 23\% igen Natronlauge soll das Hydrat $\mathrm{NaOH} \cdot 7$ aq vorliegen.

Offenbar sind die den Kurvenzügen zugrunde liegenden Vorgänge doch komplizierter, als daß aus diesen Kurven eine Gültigkeit der in Frage stehenden Be-

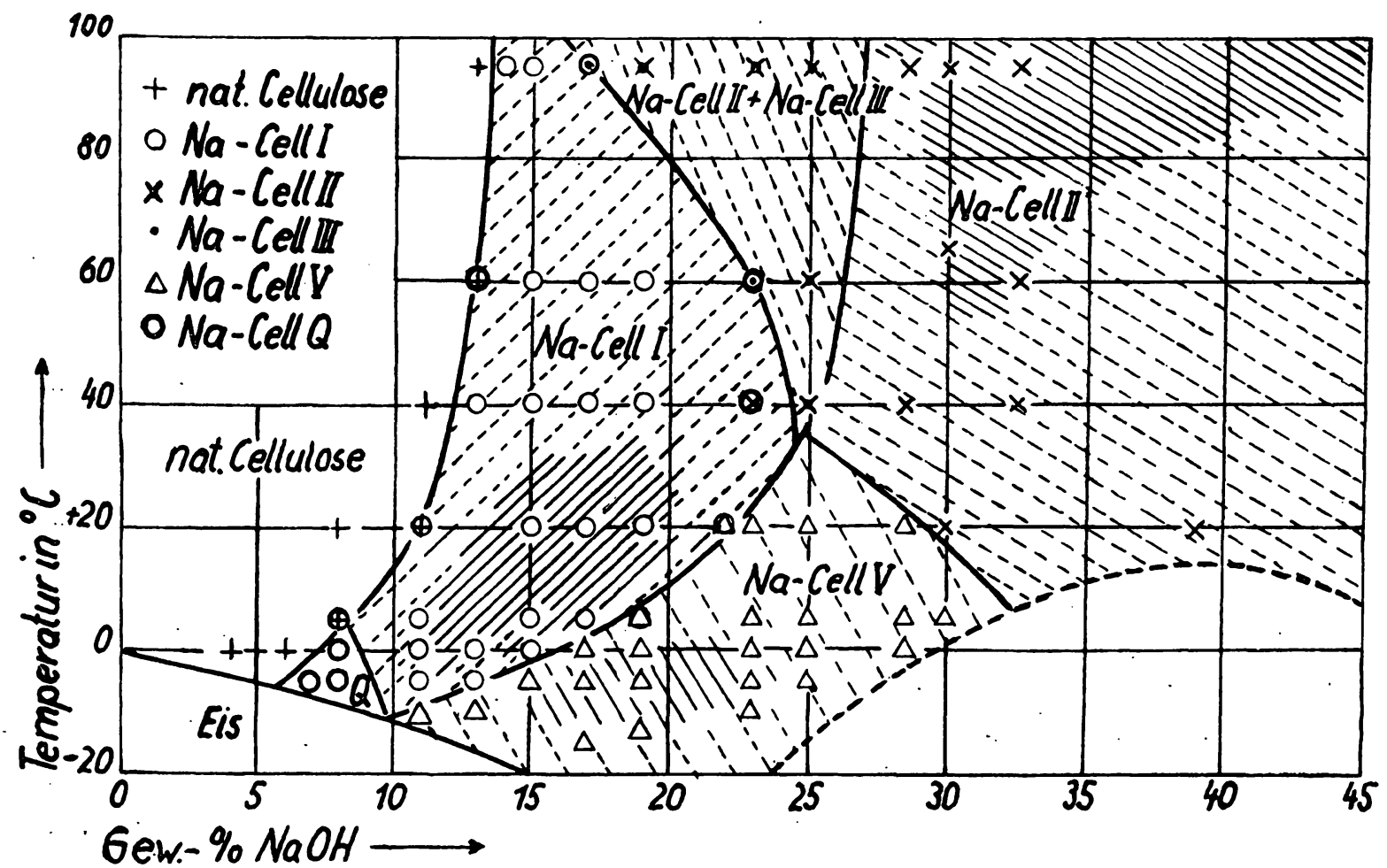

Abb. 3. Bildungsbereiche der verschiedenen Natroncellulosen in Abhängigkeit von NaOH-Konzentration und Temperatur nach $\mathrm{K}$. Hess und Mitarbeiter (3).

Die ausgezogene Schraffierung kennzeichnet die Gebiete, in denen die betreffende Natroncellulose optimal gebildet wird 
ziehung abgelesen werden könnte. Jedenâalls sind die Kurvenzüge zwanglos mit Erscheinungen in Zusammenhang zu bringen, die für eine Erklärung näher liegen als die Annahme von besonderen "Ordnungszuständen" der Natronlaugen, für die die Anwesenheit verschiedener Hydrate unter den von R. Bartunek gewählten Bedingungen $\left(20^{\circ} \mathrm{C}, 18-35 \mathrm{Gew} \% \mathrm{NaOH}\right.$ ) bisher nicht nachgewiesen worden sind. Gewiß ist der Hydratisierungszustand von $\mathrm{Na}^{+}-$und $\mathrm{OH}^{-}-$Ionen in der Natronlauge wie bei allen Elektrolytlösungen von der Konzentration abhängig. Es existiert dann jedoch keine scharfe Grenze zwischen den einzelnen Hydratisierungsstufen, vielmehr sind über den ganzen Konzentrationsbereich eine größere Anzahl verschieden stark hydratisierter Ionen nebeneinander vorhanden. Lediglich die Häufigkeit der Hydratisierungsstufen ändert sich mit der Konzentration, aber stetig und gleichmäßig ohne Diskontinuitäten.

Wenden wir uns nun der in Gegenwart von Cellulose erhaltenen Lösungskurve II $\mathrm{zu}$, so ist $\mathrm{zu}$ berücksichtigen, daß sich unter den von B artu nek gewählten Reaktionsbedingungen $\left(20^{\circ} \mathrm{C}\right)(0-44 \% \mathrm{NaOH})$ entsprechend $\mathrm{Abb} .3$ drei verschiedene Natroncellulosen bilden:

$\mathrm{Na}-\mathrm{Cell} \mathrm{I}$ ab etwa $10 \% \mathrm{NaOH}, \mathrm{Na}-\mathrm{Cell} \mathrm{V}$ zwischen etwa etwa 23 und $28 \%$ und $\mathrm{Na}$-Cell II etwa oberhalb $30 \%$; unterhalb $10 \% \mathrm{NaOH}$ bildet sich gittergeordnete Alkali-Cellulose überhaupt nicht oder nur in einem sehr geringen Umfang.

Jede dieser Alkalicellulosen hat, wie durch die in Abb. 4 wiedergegebenen reaktionskinetischen Versuche geprüft wurde, andere Reaktionskonstanten für die Umsetzung mit $\mathrm{CS}_{2}$, die auch dann noch verschieden bleiben, wenn die Fasern mit verdünnten Laugen

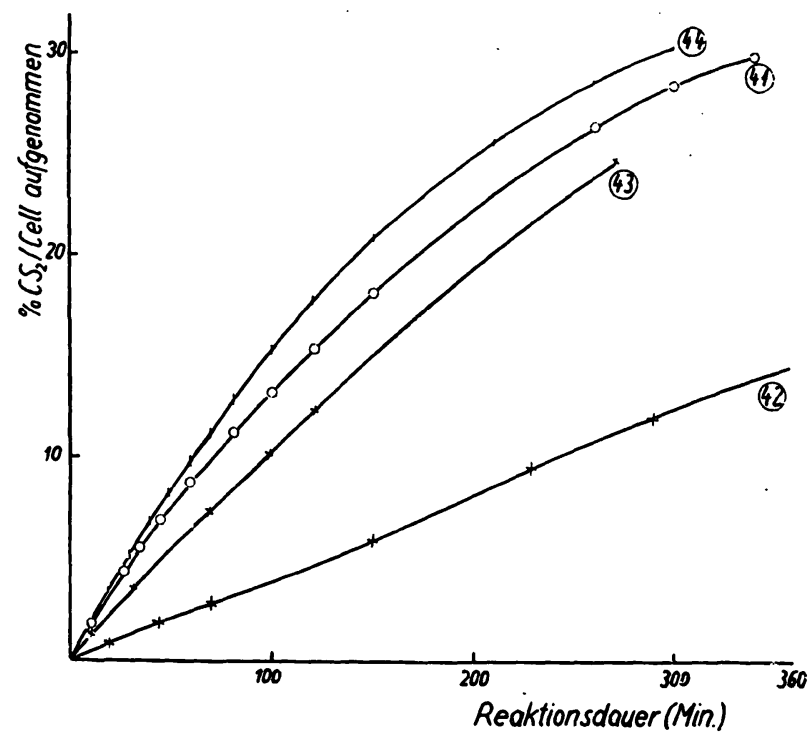

Abb. 4. Zeitliche Verfolgung der Xanthogenierung von NaCell I, Na-Cell V und Na-Cell II (Preßfaktor nach dem Tauchen 2,8; $\mathrm{CS}_{2}$ gasförmig). (Nach Versuchen von $\mathrm{H}$. Grotjahn.)

\begin{tabular}{|c|c|c|}
\hline $\begin{array}{l}\text { Versuchs- } \\
\mathrm{Nr} .\end{array}$ & $\begin{array}{l}\text { Kennzeichnung } \\
\text { der Alkalicellulose }\end{array}$ & $\begin{array}{c}\text { Analyse } \\
\text { der Alkalicellulose }\end{array}$ \\
\hline $\begin{array}{l}42 \\
43\end{array}$ & $\begin{array}{l}\mathrm{Na}-\text { Cell II } \\
\text { Na-Cell II } \\
\text { rückgetaucht }\end{array}$ & $\begin{array}{l}33,4 \% \text { Cell., } 27,5 \% \mathrm{NaOH} \\
33,1 \% \text { Cell., } 14,1 \% \mathrm{NaOH}\end{array}$ \\
\hline 4I & $\mathrm{Na-Cell} \mathrm{V}$ & $32,4 \%$ Cell., $14,1 \% \mathrm{NaOH}$ \\
\hline 44 & Na-Cell I & $30,1 \%$ Cell., $13,9 \% \mathrm{NaOH}$ \\
\hline
\end{tabular}

22 Holzforschung Bd. 9. Heft 6 unter solchen Bedingungen zurückgetaucht werden, daß die betreffende Gitterordnung der Alkalicellulose dabei nicht verändert wird, sondern nur die Konzentration der anhaftenden Lauge.

Diese Versuchsergebnisse bedeuten für die Versuchsbedingungen der Kurve II in Abb. 2, daß in diese die verschieden weit fortgeschrittene Xanthogenierung eingeht, die bei $20^{\circ} \mathrm{C}$ auch noch nach $360 \mathrm{Min}$. bei $\mathrm{Na}-$ Cell I nur zu $\% \mathrm{CS}_{2}=15$, bei Na-Cell V zu \% $\mathrm{CS}_{2}=28$, bei Na-Cell II zu $\% \mathrm{CS}_{2}=32$ führt, wenn die Fasern mit einem Preßfaktor von 2,8 gasförmigem $\mathrm{CS}_{2}$ ausgesetzt werden. Unter den von Bartunek gewählten Bedingungen dürfte die Reaktion infolge der Diffusionsverhältnisse (Umwälzzylinder) bei den verschiedenen Alkalicellulosen noch weniger weit fortgeschritten sein. Dies wirkt sich dann in dem Sinne aus, $\mathrm{da} B$ mit zunehmender Laugenkonzentration entsprechend der für die einzelnen Alkalicellulosen in der Reihenfolge Na-Cell I, Na-Cell V und Na-Cell II abnehmenden Xanthogenierungsgeschwindigkeiten abnehmende Mengen Cellulose in Lösung gehen.

Eine weitere Erscheinung, die außerhalb der Bildung der Alkalicellulose und ihrer Xanthogenierung liegt und die für Kurve II sehr zu beachten ist, ist die mit zunehmender Laugenkonzentration abnehmende Löslichkeit des Xanthates. Die von uns bisher nur für

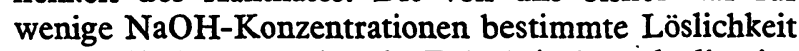
von Cellulosexanthat in Tab. 1 (vgl. auch die eingezeichneten Punkte in Abb. 2) läßt eindeutig erkennen, daß diese entsprechend dem absteigenden Ast der Kurve II in Abb. 2 mit der Laugenkonzentration: in einem solchen. Maße abnimmt, daß man den. Eindruck gewinnt, daß tatsächlich. kaum etwas anderes als diese Löslichkeitsabnahme durch den absteigenden Ast der Kurve gemessen worden sein dürfte.

Wir sind der Auffassung, daß für den Kurvenverlauf neben der Löslichkeit des gebildeten Cellulosexanthogenats aber auch noch der im gleichen. Sinne wirkende Einfluß der im vorangehenden Abschnitt behandelten Art in gewissem Umfang zu berücksichtigen ist.

Auch für ein Verständnis der Kurve I der Abb. 2, die die Abhängigkeit der Trithiocarbonatbildung aus $\mathrm{CS}_{2}$ in steigenden Konzentrationen der Natronlauge darstellt, bedarf es nicht der Annahme von besonderen Ordnungszuständen in den Natronlaugen. In diesem Falle ist ebenfalls die Kinetik bei den von Bartunek gewählten Reaktionsbedingungen zu berücksichtigen. Nach Versuchen von H. Grotjahn (4) besteht entsprechend Abb. 5 eine erhebliche Abhängigkeit der Geschwindigkeitskonstanten für diese Reaktion von der

Tab. I. Quellung und Lösung von Xanthat in Abhängigkeit von der $\mathrm{NaOH}-$ Konzentration ( $\mathrm{I} \%$ ige Lösungen bezogen auf Cellulose)

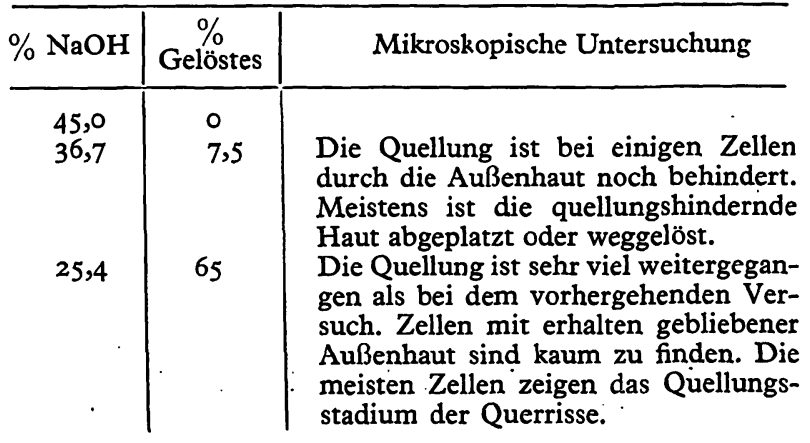




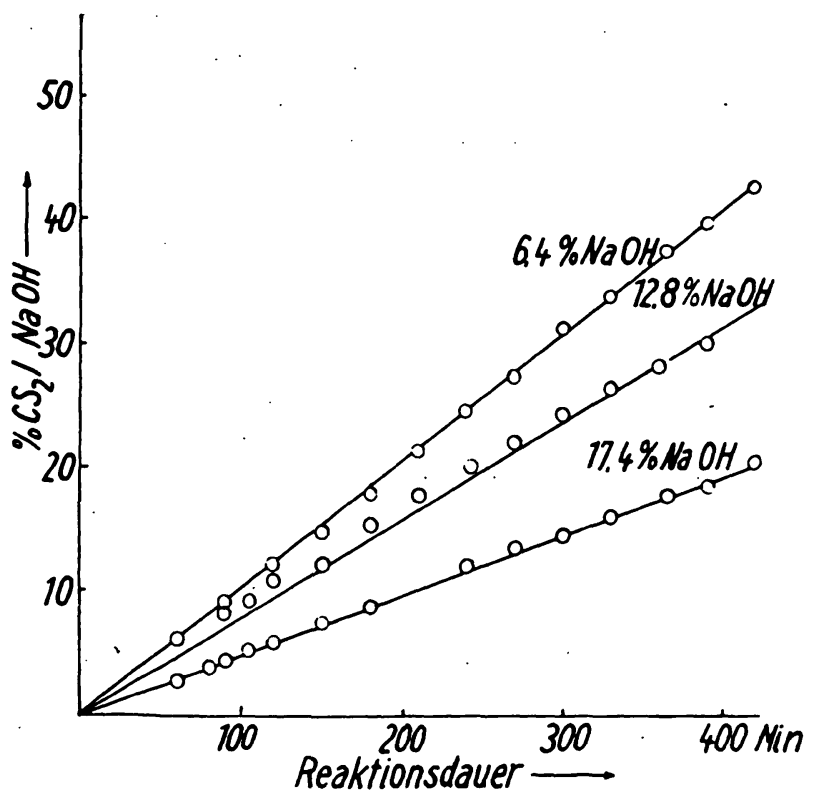

Abb. 5. Geschwindigkeit der Umsetzung von $\mathrm{CS}_{2}$ mit Natronlauge bei verschiedenen $\mathrm{NaOH}-K o n z e n t r a t i o n e n, 23,4^{\circ} \mathrm{C}$, $\mathrm{CS}_{2}$-Anfangsdruck 240 Torr, Volumen des Reaktionsraumes $1300 \mathrm{ccm}$, Menge Natronlauge $4 \mathrm{~g}$

Na-Konzentration, die nach $400 \mathrm{Min}$. bei $6,4 \%$ iger Natronlauge etwa $45 \% \mathrm{CS}_{2}$; bei $12,8 \%$ iger Lauge etwa $32 \%$ und bei $17,4 \%$ iger Lauge erst $20 \% \mathrm{CS}_{2}$ umgesetzt hat.

Nun sind zwar die Versuchsbedingungen in beiden Fällen nicht streng vergleichbar; man kann aber gewiß sein, daß unter den Versuchsbedingungen von R. B artunek (Umwälzverfahren, flüssig/flüssig gegenüber flüssig [bei feiner Verteilung]/gasförmig) nach $360 \mathrm{Min}$. nach unserer Auffassung auch noch sehr verschiedene
Reaktionsfortschritte gemessen sind und keine verschiedenen Endgleichgewichte, die durch verschiedene Reaktionsmechanismen bedingt werden.

Ergeben sich in dieser Weise einige Faktoren für den Verlauf im absteigenden Ast der Kurven von Bartunek, die weniger mit einer Beeinflussung der Reaktionen zwischen $\mathrm{NaOH}$ und Cellulose bzw. Schwefelkohlenstoff durch verschiedene Hydratationszustände des Natriumhydroxyds in den Natronlaugen zusammenhängen als vielmehr mit den Eigenschaften der $\mathrm{Re}-$ aktionsprodukte selbst (verschiedene Kinetik der im untersuchten Konzentrationsbereich sich bildenden verschiedenen Natroncellulosen, abnehmende Löslichkeit des Xanthats mit zunehmender Konzentration der Natronlauge), so soll jetzt erörtert werden, ob die beobachteten Wendepunkte selbst für die Wirkung verschiedener Hydratationsgrade angesprochen werden können. Wir beschränken uns dabei auf die Umsetzung zwischen $\mathrm{NaOH}$ und $\mathrm{CS}_{2}$ in der Schwingmühle, bei der der Wendepunkt sehr ausgeprägt ist; für einen exakten Vergleich mit der Umsetzung in Gegenwart von Cellulose fehlt die Versuchsreihe in der Schwingmühle.

Geht man davon aus, daß sich als Reaktionsendprodukte zwischen $\mathrm{CS}_{2}$ und $\mathrm{NaOH}$ Trithiocarbonat, $\mathrm{Na}$ triumcarbonat und Schwefelnatrium gemäß folgender Bruttogleichung bilden:

$$
{ }_{2} \mathrm{CS}_{2}+6 \mathrm{NaOH}=\mathrm{Na}_{2} \mathrm{CS}_{3}+\mathrm{Na}_{2} \mathrm{~S}+\mathrm{Na}_{2} \mathrm{CO}_{3}+3 \mathrm{H}_{2} \mathrm{O}
$$

dann ist zu erwarten, daß sich bei dem verwendeten Überschuß an $\mathrm{CS}_{2}$ und bei genügend langer Reaktionsdauer die jeweils vorhandene Natronlauge vollständig entsprechend dieser Gleichung umsetzt. Nach den Angaben ivon Bartunek ist dies gemäß Abb. 6 bis etwa $10 \%$ ige Natronlauge auch der Fall. Darüber hinaus nimmt der Verbrauch an Natronlauge zunächst langsam und oberhalb $23 \%$ schneller ab.

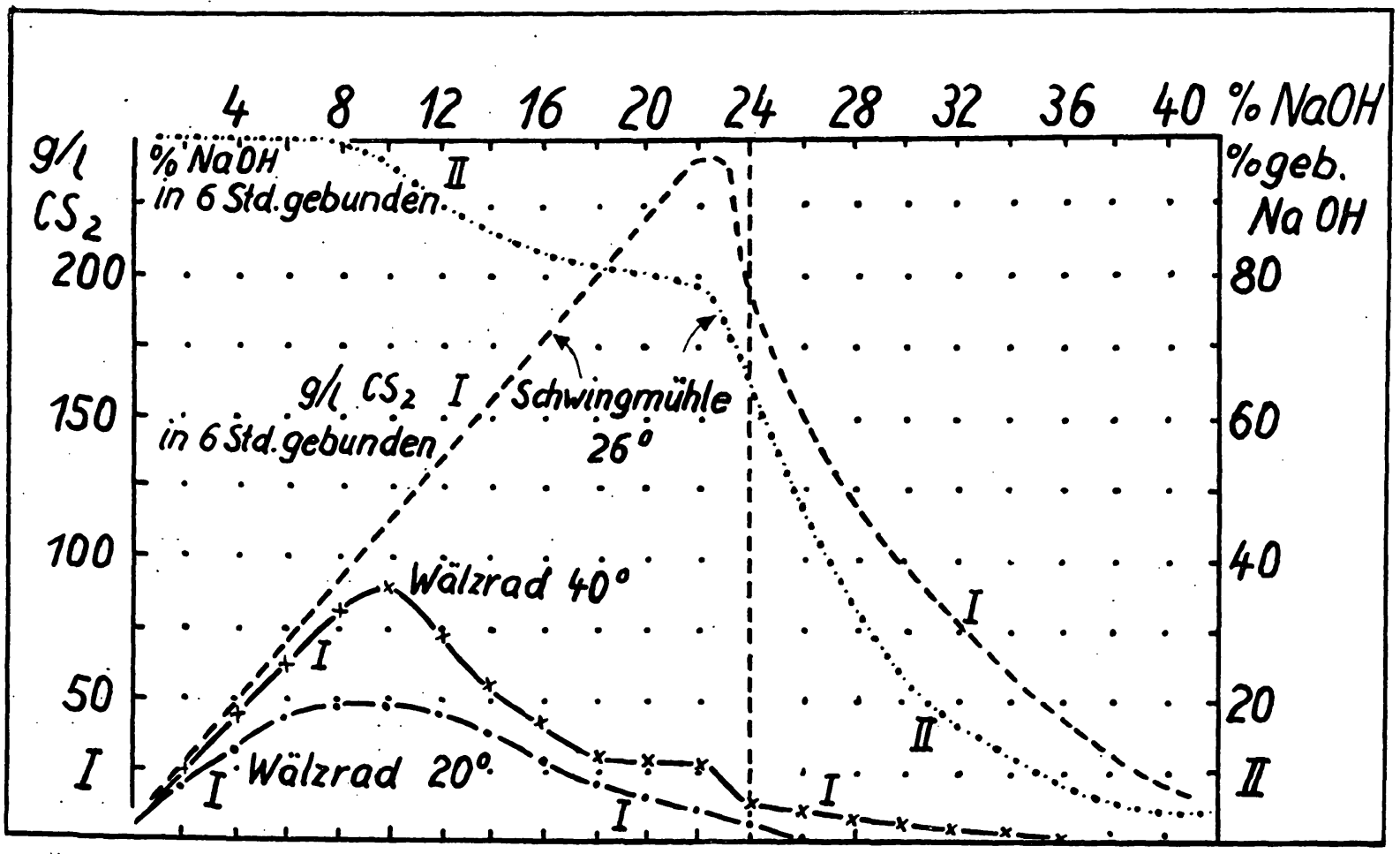

Abb. 6. Kurve I $\mathrm{CS}_{2}$-Bindung in $\mathrm{g} / 1$ durch $\mathrm{NaOH}$ steigender Konz. bei Behandlung in der Schwingmühle; Kurve II NaOHVerbrauch in \% Ausgangs- $\mathrm{NaOH}$ (nach Bartune $\mathrm{k}$ ) 
Nun hat H. Grotjahn nachgewiesen, daß die Reaktionskonstante für die Umsetzung mit zunehmender $\mathrm{NaOH}-$ Konzentration sehr erheblich abnimmt (vgl. $A b b .5)$ und daß bei den höheren Laugenkonzentrationen die von Bartunek gewählte Reaktionsdauer von 360 Minuten nicht ausreicht, um alles $\mathrm{NaOH}$ umzusetzen. Wenn bei Lösungen oberhalb einer gewissen Konzentration (etwa 23\%) der Abfall der verbrauchten $\mathrm{NaOH}$ größer wird, so ist zu berücksichtigen, daß bei höheren $\mathrm{NaOH}-\mathrm{Konzentrationen} \mathrm{die} \mathrm{Umsatzgeschwin-}$ digkeit vor allem von Trithiocarbonat immer stärker im Sinne einer Reaktionsverzögerung wirksam werden dürfte, so daß der in Frage stehende Kurvenzug in erster Linie durch die wechselnde Reaktionskinetik des Systems gedeutet werden kann. Das in den gezeigten Kurven der $\mathrm{Abb} .2$ auftretende Maximum läßt sich dementsprechend auch in einfacher Weise durch die Abnahme der Löslichkeit des $\mathrm{CS}_{2}$ mit steigendem Elektrolytgehalt erklären, ohne daß die Annahme einer sonstigen speziellen Änderung in der Reaktionsweise nötig ist. Daß dabei Hydratationsgleichgewichte der Natronlauge selbst eine Rolle spielen können, wird nicht bestritten; zu berücksichtigen sind dann aber auch die starken wasserbindenden Kräfte des äußerst hygroskopischen Trithiocarbonats und auch des Schwefelnatriums. Im übrigen spielen unter den Bedingungen von Bartunek bei der Kinetik Diffusionseffekte eine übergeordnete Rolle.

Zusammenfassend kommen wir also zu dem Schluß, daß auch der Wendepunkt der $\mathrm{CS}_{2}-\mathrm{NaOH}$-Kurve ohne weiteres nicht geeignet ist, um einen Wechsel in der Reaktionsweise („Reaktions-Wendepunkt") zwischen $\mathrm{NaOH}$ und $\mathrm{CS}_{2}$ zu begründen.

\section{Schlußbetrachtung}

Wir betonen, da $\dot{B}$ sich die tatsächlichen Verhältnisse bei der Reaktion im System Cellulose $/ \mathrm{NaOH} / \mathrm{H}_{2} \mathrm{O} / \mathrm{CS}_{2}$ im Rahmen der in den Jahren 1951/54 durchgeführten Untersuchungen durchaus befriedigend übersehen lassen und daß sich dies namentlich auch im Hinblick auf die von R. Bartunek in seiner Einleitung unter „Kenntnislücken“ herausgestellten Fragen bezieht:

1. Wie ist die Cellulose/ $\mathrm{NaOH}$-Verbindung aufgebaut, welche mit $\mathrm{CS}_{2} \mathrm{zu}$ reagieren vermag und so die Grundlagen der ganzen Viskose-Industrie bildet?

2. Warum ist der $\mathrm{NaOH}-\mathrm{Konzentrationsbereich} \mathrm{der}$ Tauchlauge zur optimalen Viskosierung so eng?

3. Warum lösen sich xanthogenierte Fasern gerade bei 8-bis $9 \%$ iger Konzentration am besten?

$\mathrm{Zu}$ 1) Die erste Frage erledigt sich ohne weiteres durch die Strukturbestimmung an Natroncellulose und an Xanthat, wie sie in der Abhandlung $\mathrm{K}$. Hess, H. Kiessig, W. Koblitz (5), S. 706, quantitativ durchgeführt worden ist.

$\mathrm{Zu}$ 2) darf darauf hingewiesen werden, daß der Konzentrationsbereich der Natronlauge für die optimale Viskosierung gar nicht so eng ist. Im übrigen spielt hier die Löslichkeit des Xanthats in der $\mathrm{NaOH}$ die entscheidende Rolle.
$\mathrm{Zu}$ 3) Diese Frage erledigt sich zum Teil durch Frage 2. Es kommen aber noch andere Faktoren hinzu, so der Grad der Gitteraufweitung des Xanthates, worüber in einer ausführlichen Studie (6) unlängst berichtet wurde.

Hinsichtlich der Abhängigkeit der Löslichkeit des Xanthats von der $\mathrm{NaOH}-\mathrm{Konzentration}$ sind neben der hydrolytischen und elektrochemischen Dissoziation der Natriumverbindungen die Hydratationszustände der Natronlauge und des Xanthats zu berücksichtigen.

In dieser Beziehung stimmen wir der grundsätzlichen Auffassung über eine Bedeutung der Hydratationszustände der gelösten Komponenten des Systems zu. Man wird sich darüber Klarheit verschaffen können, wenn man von dem Xanthat selbst ausgeht und seine Löslichkeit zu den mit besonderen Methoden zu ermittelnden Hydratationszuständen der Natronlaugen unter den Lösebedingungen in Beziehung zu stellen versucht.

\section{Zusammenfassung}

Die von $R$. Bartunek verschiedentlich gegebene $\mathrm{Zu}-$ ordnung der Reaktionsweise zwischen Schwefelkohlenstoff und dem System $\mathrm{NaOH} / \mathrm{H}_{2} \mathrm{O} /$ Cellulose zu verschiedenen Hydraten von $\mathrm{NaOH}$ in den verwendeten Laugen wird kritisch besprochen. Die von Bartunek als Argument für diese Zuordnung durchgeführten Löslichkeitsversuche lassen sich ohne weiteres durch die von der $\mathrm{NaOH}$-Konzentration abhängige Löslichkeit des gebildeten Cellulose-Xanthats erklären. Auch sind die Reaktionsverhältnisse bei den von Bartunek gewählten Versuchsbedingungen durch die Bildung der gittermäßig verschiedenen Natroncellulosen unduchsichtig, die gegenüber $\mathrm{CS}_{2}$ eine verschiedene Reaktionskonstante haben.

\section{Summary}

The coordination of the reaction method between carbon disulphide and the system $\mathrm{NaOH} / \mathrm{H}_{2} \mathrm{O} /$ cellulose to various hydrates of $\mathrm{NaOH}$ in the lyes used, as repeatedly given by $R$. Bartunek, is critically discussed. The solubility tests made by R. Bartune $k$ as an argument for this coordination are to be easily declared by the solubility of the formed cellulose xanthate which is dependent on the $\mathrm{NaOH}$-concentration. The reaction proportions with the test conditions, chosen by Bartunek, are also unclear on account of the formation of the differing lattice-like sodium oxide celluloses which have a different reaction constant towards $\mathrm{CS}_{2}$.

\section{Literatur}

(I) R. Bartunek, Ztschr. Angew. Chem. 63, 484 (1951); Das Papier 7, I53 (1953); Koll. Ztschr. 133, I25 (1954).

(2) C. Trogu s, Cellulose-Chemie 15, 104(1934); vgl. dazu auch die neueren Untersuchungen von J. Chédin, Journ. Chim. Phys. 49, I09 (1952); Koll. Ztschr. 125, 65 (1952).

(3) H. Sobue, H. Kiessig und K. Hess, Ztschr. phys.'Chrm. (B) 43, 309 (1939).

(4) H. Grotjahn, Ztschr. f. Elektrochem. 57, insbes. S. 313 (I953).

(5) K. Hess, H. Kiessig, W. Koblitz, Ztschr. Elektrcchem. 55,697(I95I); H. Grotjahn, ebenda 57,305 (1953).

(6) W. Koblitz, H. Kiessig und K. Hess, Ztschr. Elektrochem. 58, 872 (I954). 